\title{
Infinite Parametric Families of Irreducible Polynomials with a Prescribed Number of Complex Roots
}

\author{
Catalin Nitica $^{1}$, Viorel Nitica ${ }^{2}$ \\ ${ }^{1}$ Technical College Dimitrie Leonida, Bucharest, Romania \\ ${ }^{2}$ Department of Mathematics, West Chester University, West Chester, USA \\ Email: catalin@catnit.ro,vnitica@wcupa.edu
}

How to cite this paper: Nitica, C. and Nitica, V. (2019) Infinite Parametric Families of Irreducible Polynomials with a Prescribed Number of Complex Roots. Open Journal of Discrete Mathematics, 9, 1-6. https://doi.org/10.4236/ojdm.2019.91001

Received: August 26, 2018

Accepted: December 14, 2018

Published: December 17, 2018

Copyright (c) 2019 by authors and Scientific Research Publishing Inc. This work is licensed under the Creative Commons Attribution International License (CC BY 4.0).

http://creativecommons.org/licenses/by/4.0/

\begin{abstract}
In this note, for any pair of natural numbers $(n, k), n \geq 3, k \geq 1$, and $2 k<n$, we construct an infinite family of irreducible polynomials of degree $n$, with integer coefficients, that has exactly $n-2 k$ complex non-real roots if $n$ is even and has exactly $n-2 k-1$ complex non-real roots if $n$ is odd. Our work generalizes a technical result of R. Bauer, presented in the classical monograph "Basic Algebra" of N. Jacobson. It is used there to construct polynomials with Galois groups, the symmetric group. Bauer's result covers the case $k=1$ and $n$ odd prime.
\end{abstract}

\section{Keywords}

İrreducible Polynomial, Complex Roots, Real Roots, Galois Theory

\section{Introduction}

\subsection{Here We Recall a Couple of Basic Facts and State Our Main Result}

Let $\mathbb{Z}$ denote the set of integers and let $\mathbb{Z}[\mathrm{X}]$ denote the ring of polynomials with integer coefficients. A polynomial $f$ over a field or a ring is called irreducible if it cannot be factored as a product of two non-constant polynomials with coefficients in that field. A criterion to check irreducibility over the rational numbers is Eisenstein irreducibility criterion [Exercise 2, page 127] [1], which states that, given $f \in \mathbb{Z}[\mathrm{X}]$, if there exists a prime $p$ that divides all coefficients of $f$ except the leading coefficient and if $p^{2}$ does not divide the free term, then $f$ is irreducible over the rationales. It is also irreducible over the integers, unless all its coefficients have a nontrivial factor in common. 


\subsection{Our Main Result Is the Following Theorem}

Theorem 1. For any pair of natural numbers $(n, k), n \geq 3, k \geq 1$, and $2 k<n$, there exists an infinite parametric family of irreducible polynomials in $\mathbb{Z}[X]$ of degree $n$, which have exactly $n-2 k$ complex non-real roots if $n$ is even and exactly $n-2 k-1$ complex non-real roots if $n$ is odd.

Remark 1 . The case $k=0$ can also be easily covered. If $n$ is even, consider $P_{\lambda}(x)=x^{n}+\lambda, \lambda>1$ prime, which is irreducible due to Eisenstein criterion and has no real roots. If $n$ is odd, consider $P_{\lambda}(x)=x^{n}+\lambda, \lambda>1$ prime, which is irreducible due to Eisenstein criterion and has exactly one real root.

Our result generalizes a technical result of the American mathematician R. Bauer which is presented in the classical monograph "Basic Algebra" of N. Jacobson [see the proof of Theorem 8, page 107] [1]. The result of Bauer is used to construct polynomials with prescribed Galois groups, more precisely the symmetric group. Bauer's result covers the case where $k=1$ and $n$ is an odd prime from Theorem 1.

\section{The Basic Construction}

Observe that if $n=3, k=1$, the polynomial $f=x^{3}+2$ is irreducible and has exactly two non-real roots. Therefore in what follows we can assume that $n \geq 4$.

From now on $n, k$ are integers such that $n \geq 4, k \geq 1,2 k<n$. In order to simplify the notation, we introduce the variable $m=n-2 k$ if $n$ is even and $m=n-2 k-1$ if $n$ is odd. In both cases $m$ is even.

We define

$$
a_{k}=2 k, a_{k-1}=2(k-1), a_{k-2}=2(k-2), \cdots, a_{2}=4, a_{1}=2 .
$$

If $n$ is even define

$$
g(x)=\left(x-a_{k}\right)\left(x-a_{k-1}\right) \cdots\left(x-a_{1}\right)\left(x+a_{1}\right) \cdots\left(x+a_{k-1}\right)\left(x+a_{k}\right)
$$

and if $n$ is odd define

$$
g(x)=\left(x-a_{k}\right)\left(x-a_{k-1}\right) \cdots\left(x-a_{1}\right) x\left(x+a_{1}\right) \cdots\left(x+a_{k-1}\right)\left(x+a_{k}\right) .
$$

In both cases define

$$
f_{\lambda}(x)=\left(x^{m}+\lambda\right) g(x)
$$

where $\lambda>1$ is an odd positive integer of size to be determined later.

The polynomial $g(x)$ has exactly $n-m$ real roots. It follows from Rolle's Theorem that $g(x)$ has $n-m-1$ points of local extrema:

$$
\begin{gathered}
e_{1}<e_{2}<\cdots<e_{n-m+1}, \\
E_{1}=g\left(e_{1}\right), E_{2}=g\left(e_{2}\right), \cdots, E_{n-m-1}=g\left(e_{n-m-1}\right) .
\end{gathered}
$$

The points of local extrema alternate between local maxima and local minima. On all intervals

$$
\left(-\infty, e_{1}\right),\left(e_{n-m-1},+\infty\right),\left(e_{i}, e_{i+1}\right), 1 \leq i \leq n-m-1,
$$

the polynomial $g(x)$ is strictly monotonic and the derivative $g^{\prime}(x)$ has con- 
stant sign. Otherwise, $g^{\prime}(x)$ would have other zeroes besides $\pm a_{k}$, so overall more then $n-m-1$, in contradiction with the fact that it is a polynomial of degree $n-m-1$.

If $n$ is odd, the minimum of the absolute value of the polynomial $g(x)$ in the points of local extrema is larger then the minimum of the absolute value of $g(x)$ in the odd integers in the interval $[-2 k, 2 k]$. If $n$ is even, the minimum of the absolute value of the polynomial $g(x)$ in the points of local extrema is larger then the minimum of the absolute value of $g(x)$ in the odd integers in the interval $[-2 k, 2 k]$ and in zero. In the first case the minimum is $(2 k) !(2 k) ! \geq 4>2$ and in the second case the minimum is $(2 k+1) !(2 k-1) ! \geq 6>2$. If we denote $\tilde{E}_{i}=f\left(e_{i}\right), 1 \leq i \leq n-m-1$, then

$$
\left|\tilde{E}_{i}\right| \geq 3,1 \leq i \leq n-m-1
$$

Lemma 1. If $g^{\prime}(x)$ has constant sign, strictly positive or strictly negative, on an interval $(a, b)$ from (1), then for any $\delta>0$ such that $(a+\delta, b-\delta) \subset(a, b)$, there exists $\lambda>0$ such that $f_{\lambda}^{\prime}(x)$ has the same sign with $g^{\prime}(x)$ on the interval $(a+\delta, b-\delta)$.

Proof. We first considered the case when $a$ and $b$ are finite numbers and $g^{\prime}(x)>0, x \in(a, b)$. Let

$$
M_{1}=\max _{x \in[a, b]}\left|m x^{m-1} g(x)\right|, M_{2}=\min _{x \in[a+\delta, b-\delta]} g^{\prime}(x)>0, c=\max \{|a|,|b|\} .
$$

One has $f_{\lambda}^{\prime}(x)=m x^{m-1} g(x)+\left(x^{m}+\lambda\right) g^{\prime}(x) \geq-M_{1}+M_{2}\left(-c^{m}+\lambda\right)$ and for $\lambda>\frac{M_{1}+1}{M_{2}}+c^{m}$ the right member of the last equation is greater than 1 for $x \in(a+\delta, \mathcal{B}-\delta)$.

Assume now that $g^{\prime}(x)<0, x \in(a, b)$.

Let $M_{1}=\max _{x \in[a, b]}\left|m x^{m-1} g(x)\right|, M_{2}=\min _{x \in[a+\delta, b-\delta]} g^{\prime}(x)<0$, $c=\max \{|a|,|b|\}$.

One has $f_{\lambda}^{\prime}(x)=m x^{m-1} g(x)+\left(x^{m}+\lambda\right) g^{\prime}(x) \leq M_{1}+M_{2}\left(c^{m}+\lambda\right)$. Then for $\lambda>\frac{-M_{1}-1}{M_{2}}-c^{m}$ the right member of the last equation is less than -1 when $x \in(a+\delta, b-\delta)$.

If $(a, b)=(-\infty, b)$, then $b<e_{1}$. In particular, $b<0$. Also, $g^{\prime}(x)>0, x \in(a, b)$, if $n$ is odd and $g^{\prime}(x)<0, x \in(a, b)$, if $n$ is even. Assume that $n$ is odd. Then $m x^{m-1} g(x)$ is a polynomial of even degree that can be negative only on a finite interval $(c, d) \subset(-\infty, b)$. On $(a, b) \backslash(c, d)$ one has $f_{\lambda}^{\prime}(x)>0$ if $\lambda>0$. We show that $g^{\prime}(x)>0$ on $[c, d]$. Define

$$
M_{1}=\max _{x \in[c, d]}\left|m x^{m-1} g(x)\right|, M_{2}=\min _{x \in[c, d]} g^{\prime}(x)>0 \text {. }
$$

One has the estimates:

$$
f_{\lambda}^{\prime}(x)=m x^{m-1} g(x)+\left(x^{m}+\lambda\right) g^{\prime}(x) \geq-M_{1}+\left(|b|^{m}+\lambda\right) M_{2} \text {. Then for }
$$


$\lambda>\frac{M_{1}+1}{M_{2}}-|b|^{m}$ the right member of the last equation is greater than 1 when $x \in(c, d)$.

Assume now that $n$ is even. Then $m x^{m-1} g(x)$ is an odd degree polynomial which can be positive only on a finite subinterval of $(-\infty, b)$, say $(c, d)$. So $f_{\lambda}^{\prime}(x)=m x^{m-1} g(x)+\left(x^{m}+\lambda\right) g^{\prime}(x)<0, x \in(c, d)$. We show that $f_{\lambda}^{\prime}(x)<0, x \in[c, d]$. Define

$$
M_{1}=\max _{x \in[c, d]}\left|m x^{m-1 ?} g(x)\right|, M_{2}=\min _{x \in[c, d]} g^{\prime}(x)<0 .
$$

One has the estimates $f_{\lambda}^{\prime}(x)=m x^{m-1} g(x)+\left(x^{m}+\lambda\right) g^{\prime}(x) \leq M_{1}+\left(|b|^{m}+\lambda\right) M_{2}$. Then for $\lambda>\frac{-M_{1}-1}{M_{2}}-|b|^{m}$ the right member of the last equation is greater than -1 when $x \in\left(c, d^{\prime}\right)$.

If $(a, b)=(a, \infty)$, then $(a, b) \subset\left(e_{n-m-1}, \infty\right), a>0$, and $\quad g^{\prime}(x)>0$ if $x \in(a, b)$, independent of the parity of $n$. The polynomial $m x^{m-1} g(x)$ can be negative only on a finite subinterval $(a, d) \subset(a, \infty)$. If $x \in(a, b) \backslash(c, d)$, then $f_{\lambda}^{\prime}(x)=m x^{m-1} g(x)+\left(x^{m}+\lambda\right) g^{\prime}(x)>0$ if $\lambda>0$.

We show that $f_{\lambda}^{\prime}(x)>0, x \in(c, d)$. Let

$$
M_{1}=\max _{x \in[c, d]}\left|m x^{m-1} g(x)\right|, M_{2}=\min _{x \in[c, d]} g^{\prime}(x)>0 .
$$

One has the estimates:

$f_{\lambda}^{\prime}(x)=m x^{m-1} g(x)+\left(x^{m}+\lambda\right) g^{\prime}(x) \geq-M_{1}+\left(|d|^{m}+\lambda\right) M_{2}$. Then for

$\lambda>\frac{M_{1}+1}{M_{2}}-|d|^{m}$ the right member of the last equation is greater than 1 when $x \in\left(c^{\prime}, d\right)$.

We are ready to prove the main result. Consider the polynomial $h_{\lambda}(x)=f_{\lambda}(x)+2$, which has the leading coefficient equal to 1 , the free coefficient divisible by 2 , but not by 4 , and due to Viete's formulas, all the other coefficients are divisible by 4 . It follows that the coefficients of $h_{\lambda}(x)$ have no nontrivial common factor and it follows from Eisenstein criterion that $h_{\lambda}(x)$ is irreducible. To finish the proof of the main result it is enough to show that for $\lambda$ large enough $h_{\lambda}(x)$ has exactly $n-m$ real roots.

Due to the continuity of $f_{\lambda}(x)$ there exists $\delta>0$ such that for any $1 \leq i \leq n-m-1$ one has

$$
\left|f_{\lambda}(x)-\check{E}_{i}\right|<\frac{1}{4}, x \in\left(e_{i}-\delta, e_{i}+\delta\right) .
$$

Because $\left|\tilde{E}_{i}\right| \geq 3$, it follows from above that for $1 \leq i \leq n-m-1$ one has

$$
\left|f_{\lambda}(x)\right| \geq\left|\tilde{E}_{i}\right|-\left|f_{\lambda}(x)-\tilde{E}_{i}\right| \geq 3-\frac{1}{4}=\frac{11}{4}, x \in\left(e_{i}-\delta, e_{i}+\delta\right) .
$$

After choosing a smaller $\delta>0$, if needed, one can apply Lemma 1 and choose $\lambda \in \mathbb{Z}$ such that the sign of $f_{\lambda}^{\prime}(x)$ is constant, strictly positive or strictly negative, on each of the following intervals: 


$$
\begin{gathered}
\left(-\infty, e_{1}-\delta\right) \\
\left(e_{1}+\delta, e_{2}-\delta\right) \\
\left(e_{2}+\delta, e_{3}-\delta\right) \\
\cdots \\
\left(e_{n-m-3}+\delta, e_{n-m-2}-\delta\right) \\
\left(e_{n-m-2}+\delta, e_{n-m-1}-\delta\right) \\
\left(e_{n-m-1}+\delta,+\infty\right)
\end{gathered}
$$

For all the intervals above, due to (2) the image of $f_{\lambda}(x)$ contains the interval $\left(-\frac{11}{4}, \frac{11}{4}\right)$. As $f_{\lambda}(x)$ is continuous it follows that the line $y=-2$ intersects the graph of the polynomial $f_{\lambda}(x)$ over these intervals in exactly $n-m$ points, with $f_{\lambda}^{\prime}(x)$ in these points different from zero. Due to (2), outside the intervals above $h_{\lambda}(x)=f_{\lambda}(x)+2$ cannot be zero. Therefore $h_{\lambda}(x)$ has $n-m$ real roots, all simple.

\section{Conclusions}

In this paper we construct infinite parametric families of irreducible polynomials in $\mathbb{Z}[\mathrm{X}]$ with a prescribed number of complex, non-real, roots. Of some interest would be to find good estimates for the smallest good value of the parameter $\lambda$. The proof of Theorem 1 provides some rough estimates of order $O\left(\left(\frac{n}{2}\right)^{n / 2}\right)$ where $n$ is the degree of the polynomial. Numerical experiments, nevertheless, show that many times $O(n)$ is sufficient. The following examples illustrate this observation.

Example 1. Let

$$
P(x)=(x-8)(x-6)(x-4)(x-2) x(x+2)(x+4)(x+6)(x+8)\left(x^{10}+10^{10}\right)+2
$$

for $n=19, \lambda=10^{10}, k=4 . P(x)$ has 10 complex non-real roots and 9 real roots.

Example 2. Let

$$
P(x)=(x-8)(x-6)(x-4)(x-2) x(x+2)(x+4)(x+6)(x+8)\left(x^{10}+10\right)+2
$$

for $n=19, \lambda=10, k=4 . P(x)$ has 10 complex non-real roots and 9 real roots.

\section{Example 3. Let}

$$
P(x)=(x-8)(x-6)(x-4)(x-2)(x+2)(x+4)(x+6)(x+8)\left(x^{10}+10^{10}\right)+2
$$

for $n=18, \lambda=10^{10}, k=4 . P(x)$ has 10 complex non-real roots and 8 real roots.

\section{Example 4. Let}

$$
P(x)=(x-8)(x-6)(x-4)(x-2)(x+2)(x+4)(x+6)(x+8)\left(x^{10}+10\right)+2
$$

for $n=18, \lambda=10, k=4$. $P(x)$ has 10 complex non-real roots and 8 real roots. 


\section{Acknowledgements}

The second author is grateful to his colleague, Professor Shiv Gupta from West Chester University of Pennsylvania, for initiating several discussions that leaded to this paper. He is also grateful to the referee of the paper for useful comments.

\section{Conflicts of Interest}

The authors declare no conflicts of interest regarding the publication of this paper.

\section{References}

[1] Jacobson, N. (1964) Lectures in Abstract Algebra, III. Theory of Fields and Galois Theory. Springer-Verlag, Berlin. https://doi.org/10.1007/978-1-4612-9872-4 